Recepción: 01 / 03/ 2018

Aceptación: 15 / 05 / 2018

Publicación: 07 / 08 / 2018
Ciencias Económicas

Artículo Científico

\title{
Sistemas de Contabilidad con Criptomonedas: Retos para la Auditoría Pública Tradicional
}

\author{
Accounting Systems with Cryptocurrency: \\ Challenges for Traditional Public Auditing
}

Sistemas de contabilidade de criptomoedas: desafios para a auditoria pública tradicional

Dewis E. Alvarez-Pincay ${ }^{\mathrm{I}}$

dewis.alvarez@unesum.edu.ec

Sandra P. Toala-Bozada II sandra.toala@unesum.edu.ec

Zoila M. Delgado-Gutierrez III zoila.delgado@unesum.edu.ec
José F. Peñafiel-Loor ${ }^{\mathrm{IV}}$

jose.penafiel@unesum.edu.ec

Adela del Jesus Lucio-Pillasagua V

adela.pillasagua@unesum.edu.ec

Viviana del Rocío Saltos-Buri VI

viviana.saltos@unesum.edu.ec

Correspondencia: dewis.alvarez@unesum.edu.ec

I. Licenciado en Contaduría y Auditoría, Master en Dirección Estratégica, Docente Titular Principal, Docente de la Universidad Estatal del Sur de Manabí, Jipijapa, Ecuador.

II. Ingeniera Comercial. Magister en Docencia Universitaria e Investigación educativa. Phd Administración, Docente de la Universidad Estatal del Sur de Manabí, Jipijapa, Ecuador.

III. Economista. Maestría en Contabilidad y Auditoría. Docente Contratada, Docente de la Universidad Estatal del Sur de Manabí, Jipijapa, Ecuador.

IV. Ingeniero Comercial. Magister en Gerencia Educativa. Docente Titular Principal Universidad Estatal del Sur de Manabí, Jipijapa, Ecuador.

v. Ingeniera en Contabilidad y Auditoría. Magister en Gerencia Educativa. Docente Contratada Universidad del Sur de Manabí, Jipijapa, Ecuador.

VI. Ingeniera Comercial. Magister en Contabilidad y Auditoría. Docente Contratada Universidad Estatal del Sur de Manabí, Jipijapa, Ecuador. 


\section{Resumen}

Las revoluciones tecnológicas han generado shocks en el funcionamiento de las sociedades, y es precisamente la tecnología Blockchain, quien ha puesto sobre la mesa el uso adecuado del dinero y la ética en las relaciones contractuales. Sin embargo, las ciencias contables no han podido seguirle el paso a diez años de su aparición: En primer lugar, las técnicas de contabilidad internacionales, que aún no logran un consenso de cómo registrar adecuadamente las criptomonedas en los estados financieros; y por último, el ejercicio de la auditoría tradicional, que se ve amenazada por la barrera de conocimiento informático que representan los smart contracts, cuestión contradictoria, porque la blockchain pretende ser la herramienta definitiva para cimentar las bases de la nueva auditoría cibernética.

Palabras claves: Blockchain, contabilidad, sistema de auditoria, criptomoneda, contratos inteligentes. 
Dewis E. Alvarez-Pincay; Sandra P. Toala-Bozada; Zoila M. Delgado-Gutierrez; José F. Peñafiel-Loor; Adela del Jesus Lucio-Pillasagua; Viviana del Rocío Saltos-Buri

\section{Abstract}

The technological revolutions have generated shocks in the functioning of societies, and it is precisely the Blockchain technology, which has put on the table the proper use of money and ethics in contractual relationships. However, the accounting sciences have not been able to keep up with ten years of their appearance: First, international accounting techniques, which have not yet reached a consensus on how to properly record cryptocurrencies in financial statements; and finally, the exercise of the traditional audit, which is threatened by the barrier of computer knowledge represented by smart contracts, a contradictory question, because the blockchain is intended to be the definitive tool to lay the foundations for the new cybernetic audit.

Keywords: Blockchain, accounting, auditing system, cryptocurrency, intelligent contracts. 


\section{Introducción.}

Es necesario introducir a este tema, que las revoluciones tecnológicas han venido forjando las sociedades, en su funcionamiento y en su capacidad cognitiva de mejorar los procesos de intercambio, en aras de abolir por completo la ignorancia y la indigencia ética en el campo monetario. En este sentido, las cadenas de bloques, o mejor conocidos como Blockchain, han venido para desmitificar el uso adecuado del dinero en las relaciones contractuales, dotándolas de transparencia, y a la vez de una capacidad definitiva en la disminución de los costos de transacción a los cuales tradicionalmente somos susceptibles. Históricamente el mundo ha crecido de forma exponencial gracias a las revoluciones tecnológicas de esta nueva era, entre ellos la bigdata y la inteligencia artificial. Desde 2008, luego de los acontecimientos ocurridos por la negligencia público-privado, en donde se engendró esta nueva propuesta peer-to-peer --como parte de esta revolución y logrando la eliminación de los intermediarios en el intercambio de dinero-- tenemos en nuestras manos la capacidad de aplicar una nueva tecnología de corte frugal, que no sólo dará una solución parcial a la crisis ética y económica en estos tiempos de aparente estabilidad, sino que impulsará aún más la intensidad del comercio internacional debido a que el sistema no contempla fronteras, derramando por todo el mundo una verdadera alternativa para reducir los costos a su mínima expresión.

A las luces de este descubrimiento, las ciencias sociales han venido buscando su acomodo, buscando el papel que vienen a ejercer en esta evolución, que, en definitiva, pone en amenaza la prescindibilidad de algunas técnicas tradicionales tales como la contabilidad. Dentro un mundo que cada vez más se torna digital y automatizado, es necesario debatir los nuevos retos y alcances que debe tomar la contabilidad y la auditoría empresarial en pro de seguir siendo esenciales para la eficiencia de la gestión administrativa, y del correcto funcionamiento de los procesos 
Dewis E. Alvarez-Pincay; Sandra P. Toala-Bozada; Zoila M. Delgado-Gutierrez; José F. Peñafiel-Loor; Adela del Jesus Lucio-Pillasagua; Viviana del Rocío Saltos-Buri

gerenciales y lograr de esta manera junto con la tecnología blockchain sumar esfuerzos que permitan seguir reduciendo las pérdidas monetarias inherentes a la actividad productiva.

\section{Antecedentes y definiciones básicas}

\section{¿Qué es el Bitcoin?}

El Bitcoin es una criptomoneda, a su vez, las criptomonedas constituyen una nueva especie de moneda digital, cuyas bases reposan sobre el cifrado, donde las transferencias capitales o hasta una sencilla transacción como el pago de un café son encriptadas de extremo a extremo para garantizar la transparencia y legitimidad del nexo financiero entre ambas partes involucradas. En contraste con el dinero fiduciario tradicional, las criptomonedas solo pueden obtenerse y canjearse por internet, lo que quiere decir que también su resguardo y su producción están estrechamente ligado a la web.

Como todo "dinero", las criptomonedas pueden ser transferidas y gastadas en cualquier punto geográfico sin que ello incremente los costos de mantenimiento del sistema $-\mathrm{y}$ cabe mencionar dentro de la misma comparativa - que será vigente su uso siempre y cuando las personas -sujetos de la intermediación-las acepten. Y es partiendo de ésta realidad que afirmamos que en el momento que la moneda carezca de confianza por parte de sus poseedores, su valor real se verá fuertemente deteriorado y es muy probable que esté cercana a su obsolescencia. Un ejemplo de ello, era cuando comerciaba sobre el patrón oro, donde las personas pudieron darse cuenta que el respaldo de los papeles del estado sobre las reservas de oro, dotaba de confianza al medio, porque en cierta medida restringía la emisión discrecional de dinero - un verdadero dolor de cabeza en el pasado--, convirtiéndolas en un medio de intercambio más confiable y estable, y por lo tanto se observó un incremento de su valor real. Del mismo modo, en el caso tal que el sistema colapse y a 
su vez la confianza que los usuarios depositaron en los administradores de las monedas fiduciarias desaparezcan, muy probablemente también será su caducidad. Quizás estemos viendo lo que puede ser una transición, porque las criptomonedas se perfilan como una panacea a todos los vicios del sistema monetario, como lo es por ejemplo la emisión de dinero sin control (vuelto a ser aceptado luego de la caída del patrón oro). En vista de ello, es lógico pensar que el ciclo de vida de las monedas tradicionales tiene sus días contados.

De tal manera que, atribuirle al Bitcoin todo el crédito en la solución de los conflictos económicos y éticos actuales es una falacia, dado que la realidad señala que; el Bitcoin trata de solventar los mecanismos de emisibilidad a través de un sistema descentralizado; y por otro lado, se habla de una novedosa tecnología que tiene aplicación en una infinidad de campos donde el hombre se interrelaciona con otro, intercambiando no sólo monedas, sino todo tipo información y acuerdos multilaterales prescindiendo de intermediarios, ésta es la tecnología blockchain ${ }^{1}$. De hecho, muchos conocedores del tema ignoran la funcionalidad y el alcance de la cadena de bloques, y es por ello que venimos a ofrecerle una visión más ampliada, y de sus aplicaciones en el área contable y de auditoría.

\section{La Blockchain como objeto central de la revolución del siglo XXI}

Siguiendo la misma línea investigativa Nathaniel Karp (2017), explica que la Blockchain es un registro encadenado de forma consecutiva, en él se encuentra el libro contable de los intercambios directos de criptomonedas entre usuarios, y además está conformado por los contratos inteligentes, cuestión que profundizaremos más adelante. Otro aspecto a resaltar es que toda la información almacenada y generada en la cadena de bloques están respaldadas en infinidades de

\footnotetext{
${ }^{1}$ Se recomienda una amplia lectura sobre esta tecnología en: Preukschat, A. (Coord.) (2017): Blockchain: La revolución industrial de internet. y Fintech: Lo que la tecnología hace por las finanzas.
} 
computadoras por todo el mundo, blindándola así de hackeos y sabotajes cibernéticos. Por ultimo, su algoritmo es capaz de reproducir transacciones bajo una serie de condiciones especiales, garantizando que el dinero comprometido de una transacción llegue a su destino sin percance alguno, cuestión que suprime la presencia de una autoridad central o terceras partes en las relaciones contractuales. Al mismo tiempo ésta metodología consta de tres elementos clave: una transacción, un registro de transacciones y un sistema que las comprueba y almacena la información de la transacción.

Los bloques se generan-sobre un software abierto — en la medida que la información procesada ha copado totalmente su capacidad de almacenamiento, dentro de estos bloques se resguarda información vital respecto al recorrido que ha tenido una unidad monetaria o contrato a través de todo el sistema, de hecho, brinda información sobre cuándo y en qué secuencia de bloques fue transada dicha moneda. Esta cronología de bloques es lo que viene a constituir la "cadena de bloques" la cual se replica cada segundo en servidores distribuidos en todo el mundo. (Ibíd.). Ésta viene a ser el aporte más grande al Bitcoin y de allí que surge el nacimiento de otras monedas, con características técnicas más diferenciadas.

\section{Funcionamiento de la tecnología Blockchain:}

Tomando en cuenta la explicación anterior, cualquier transacción aprobada es situada dentro de la cadena de bloques en orden de aparición, y a su vez, la información obtenida por cada una es replicada entre sus nodos (puntos de acceso a la cadena). La metodología en la que la Blockchain permite la realización transacciones, parte del algoritmo que viene a ser el factor discordante al sistema centralizado, el cual envía un mensaje encriptado con dos llaves, una de carácter público y otra privada. Si la transacción tiene lugar en una $e$-wallet que genera coincidencias con la llave que 
se mantiene en secreto mientras que ocurre lo mismo con la divulgada, esto le permitirá al poseedor de la clave secreta—el emisor — probarle al público en general que realmente la posee sin necesidad de mostrársela a nadie. Este procedimiento es utilizado siempre con la finalidad de verificar que los Bitcoins transferidos son auténticos. La clave pública permite generar fácilmente una dirección de Criptomoneda, la cual cumple la función de un número de cuenta en un banco, mientras que la clave privada permite crear transacciones pertenecientes a esa dirección. De esta manera, Bitcoin que se sea transado, quedará inmediatamente asentado en el libro mayor de datos, el cual se registra en los servidores descentralizados de la plataforma. Y es así como en el proceso de recreación de la transacción entre todos los nodos, las nuevas transacciones vienen a ser validadas por todas las transacciones anteriores que fueron efectuadas en la cadena, garantizando una operación "cero fraudes" a un tiempo record. A este último paso se atribuye el nombre de prueba de trabajo, o proof of work, siendo éste uno de los mayores logros por la comunidad Bitcoin.

\section{Proof of work:}

En suma, las claves públicas tienen la finalidad de que toda la comunidad identifique qué usuario es el que realiza la transferencia, pero las privadas son destinadas a validar que los Bitcoins intercambiados estén cumpliendo con la secuencia dispuesta en la blockchain. Esto quiere decir, justo cuando un usuario "envía" Bitcoins a otro, en realidad no está enviando la moneda, sino que se declara un traspaso en la propiedad de los Bitcoins que están registrados en la Blockchain. Así, de la correcta validación de estas transacciones es que se determina la recompensa que le es cancelada a los servidores que resolvieron este problema, mediante un sistema de proof of work que viene a ser la representación máxima de la descentralización que propone el Bitcoin, al no necesitar de un tercer sujeto en la transacción que valide y revise que los fondos se encuentren disponibles, de tal manera 
Dewis E. Alvarez-Pincay; Sandra P. Toala-Bozada; Zoila M. Delgado-Gutierrez; José F. Peñafiel-Loor; Adela del Jesus Lucio-Pillasagua; Viviana del Rocío Saltos-Buri

que, al tener la inquietud de saber el recorrido que ha tenido una unidad monetaria, solo bastará con auditar el libro mayor de la cadena.

A manera de plasmar mejor la idea de los programadores debemos ver la prueba de trabajo como un método para establecer un consenso, cuando de forma natural existen incentivos perversos para actuar de forma individual. En conclusión, La prueba de trabajo es la solución al conocido dilema del prisionero que tanto ha mencionado la teoría de juegos, es decir, la prueba de trabajo hace que sea más costosa estrategia no cooperativa para los participantes -los mineros-. (Remolina, 2017).

\section{Aspectos Legales}

\section{El problema del anonimato de las transacciones:}

Respecto a éste polémico tema, Echebarria, (2017) devela la realidad de los hechos:

...todo el mundo puede comprobar la cadena de bloques y ver el momento y circunstancias de una transacción. Esta trasparencia permite el contraste con los datos aportados por los usuarios, pero el sistema permite el seudónimo y por ello no identifica necesariamente al usuario de cada transacción. Comprobar una operación no es lo mismo que comprobar la autoría de la misma. Blockchain no exige la identificación previa de un usuario, lo identifica por una serie de códigos y una copia criptográfica con un sistema de criptografía publica de doble clave, por lo que cada usuario tiene sus claves almacenadas en un portafolio independiente que debe controlar...(p.84)

Esto quiere decir que mientras cada transacción con criptomonedas que es registrada en la cadena de bloques, sólo almacena datos como el valor de pago y las direcciones de las $e$-wallets, estas direcciones no se encuentran vinculadas a la identidad de los sujetos que participan en la 
operación, y por ello que se dice que las transacciones con bitcoins son anónimas o seudónimas. Aunque creamos que ello es ajeno al campo de la contabilidad y auditoría, en realidad no lo és, y es que la legitimación de capitales necesaria para la correcta gestión empresarial, imagínense la bochornosa posición donde la compañía se ve inmersa en un problema judicial por haber recibido un pago con criptomonedas que provinieron de una e-wallet que traficó armas de forma ilegal, generando todo un problema a nivel ético corporativo. Por lo tanto, creo que és necesario --partiendo de éste análisis- que en éste futuro hipotético, las empresas entrenen a un equipo auditor, que esté calificado para realizar radiografías a la blockchain de cada empresa, en búsqueda de irregularidades, éste tipo de acuerdos entre proveedor y cliente puede lograrse mediante un smart contract y con este instrumento se conocería la procedencia de las monedas que se reciben como parte de pago, y finalmente convertiremos la amenaza que proviene del pseudo anonimato, en una ventaja para la evolución de las prácticas contables. Luego expandiremos un poco más de que van estos contratos inteligentes.

\section{El bitcoin, la blockchain y su relación con la contabilidad}

Si vemos al pasado por un instante, parecería que las criptomondedas nacieron prácticamente de la nada, y es que lograron colarse fácilmente entre los particulares, de tal forma que hasta los propios bancos centrales han puesto su atención en el desarrollo de estas nuevas tecnologías. Al mismo tiempo, se utiliza cada vez más en la vida cotidiana para adquirir bienes y servicios, el volumen de transacciones de esta naturaleza sigue creciendo de forma acelerada y su cotización entre las más volátiles. No obstante, hay otra característica que las hace muy interesante como motivo de estudio: no existe una determinación especifica en las NIIF o los US GAAP (por sus siglas en inglés) sobre la contabilización de las criptomonedas, a pesar de que ya en estas instancias, muchas empresas están comenzando a adoptarlas como medios alternativos de 
Dewis E. Alvarez-Pincay; Sandra P. Toala-Bozada; Zoila M. Delgado-Gutierrez; José F. Peñafiel-Loor; Adela del Jesus Lucio-Pillasagua; Viviana del Rocío Saltos-Buri

intercambio. En tal sentido, vamos a develar según este reglamento en cuál de las partidas debería contabilizarse y por qué no en otras.

Actualmente hay un vacío normativo, no hay un reglamento claro para ubicar las criptomonedas en los estados financieros, haciendo cada vez más complicado la tarea de auditar la entrada de estos activos en las unidades productivas, por ende, nos adicionamos en la búsqueda del conocimiento, de la mano también de otros autores ( Kam, 2017 ; Bargiela, 2016 ; Sierra, 2017 ;Viloria, 2017 ; Menéndez \& Pérez, 2017), que en conjunto tienen las mismas inquietudes, al ver que la técnica contable debe ser un método que debe mutar para seguir a la vanguardia con las nuevas tecnologías.

Según las Normas Internacionales de Comercio, y tomando en consideracion los aportes de los autores con los que contamos, podemos llegar a una clasificacion que permita facilmente reconocerlas a nivel internacional -en la medida que las propias normativas se ajusten a estas nuevas tecnologías-, y por lo pronto, nos queda por descartar las clasificaciones con las que contamos para estos criptoactivos: Efectivo, Equivalentes de efectivo, Intangible y Existencias. 


\section{Esquema clasificación adecuada para las crypto}

\section{PROBLEMA: Calificación $\rightleftarrows$ valoración}

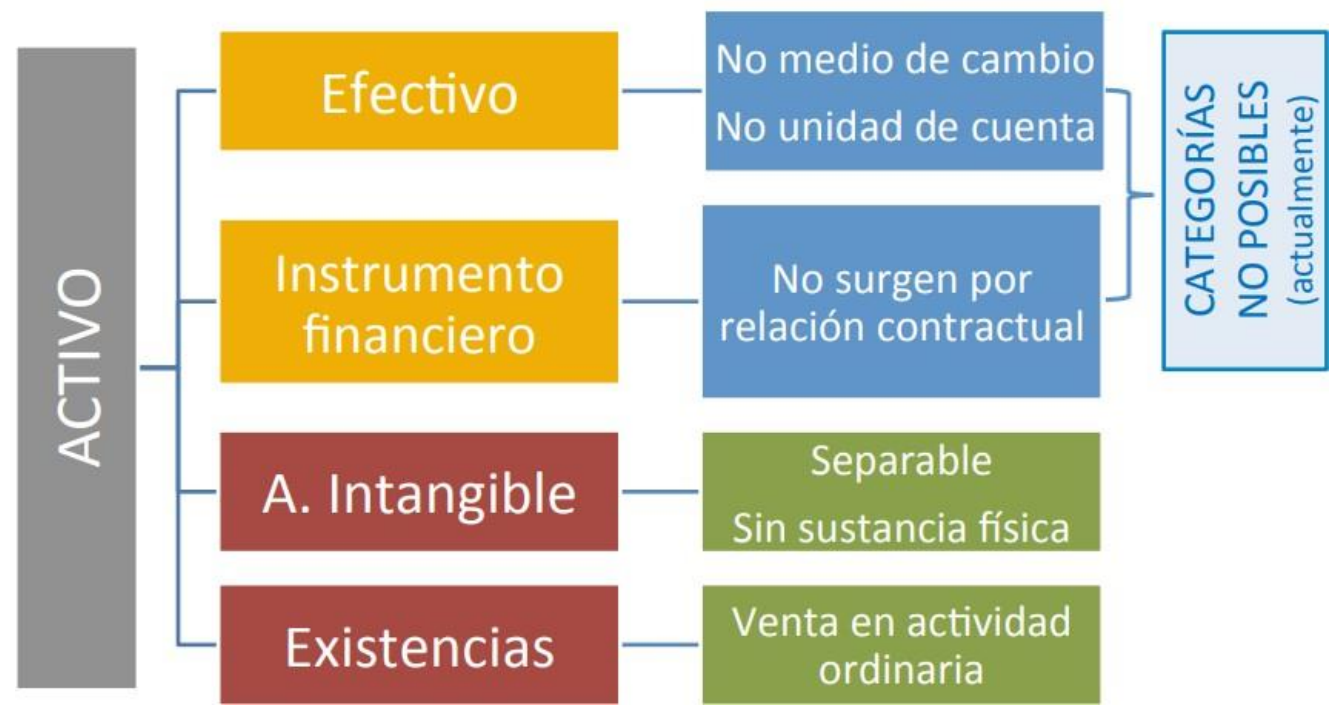

Fuente: Menéndez \& Pérez, (2017)

Efectivo y Equivalentes de efectivo:

Por un lado, la NIC 7 identifica al efectivo todo dinero que "comprende tanto la caja como los depósitos bancarios a la vista" (p.3), mientras que respecto a equivalentes de efectivo agrega:

"Los equivalentes al efectivo son inversiones a corto plazo de gran liquidez, que son fácilmente convertibles en importes determinados de efectivo, estando sujetos a un riesgo poco significativo de cambios en su valor.

Los equivalentes al efectivo se tienen, más que para propósitos de inversión o similares, para cumplir los compromisos de pago a corto plazo. Para que una inversión financiera pueda ser calificada como equivalente al efectivo, debe poder ser fácilmente convertible en una cantidad determinada de efectivo y estar sujeta a un riesgo insignificante de cambios en su valor.”(Ibíd. p.3) 
Dewis E. Alvarez-Pincay; Sandra P. Toala-Bozada; Zoila M. Delgado-Gutierrez; José F. Peñafiel-Loor; Adela del Jesus Lucio-Pillasagua; Viviana del Rocío Saltos-Buri

Por tal motivo, nos atrevemos a confirmar que no es posible clasificar las criptomonedas dentro de este renglón. Tan siquiera basta con saber que son volátiles para inmediatamente descartar esta opción, además que aún es cuestionable la alta capacidad de convertibilidad respecto a otros tipos de activos, puesto que si tomamos en cuenta que el dinero se encuentra por ejemplo, en un Exchange de criptoactivos, existen límites de retirada diarios, así como la tardanza habitual de las transferencias interbancarias de dinero fiat. Por lo tanto ésta definitivamente no es la partida adecuada.

Instrumentos financieros:

Un instrumento financiero es un contrato que da lugar, de forma simultánea, a un activo financiero para una empresa y a un pasivo financiero o instrumento de capital en otra empresa (NIC 32.11). Para dar un mayor entendimiento, en la práctica los instrumentos financieros son activos financieros para la compañía que los ha adquirido, mientras que para la empresa que los ha emitido son pasivos financieros, por ello que la norma establece que deben suceder de forma simultánea.

De hecho, Ejemplos de instrumentos financieros son: "las acciones...que constituyen el capital de una empresa; las obligaciones, ya sean simples o convertibles; los bonos o pagarés emitidos por el Estado y adquiridos por las empresas; los préstamos que una empresa concede...” (Angulo, 2004 p.246). Motivo por el cual sustraemos a las criptodivisas de ésta clasificacion, puesto que, la contraparte en la adquisicision de bitcoins ya sea por la mineria o por la transferencia de un tercero, no les representa un pasivo, dando como resultando la carencia en su caracteristica de simultaneidad que refiere la norma. 
Existencias: La norma identifica un activo como inventarios en la medida que cumpla con los siguientes criterios:

“(a) poseídos para ser vendidos en el curso normal de la explotación; 2 NIC 2

(b) en proceso de producción de cara a esa venta; o

(c) En forma de materiales o suministros, para ser consumidos en el proceso de producción o en el suministro de servicios.” (p.2-3)

De acuerdo a lo que establece el reglamento, aquí no hay un veredicto rígido, debido a que su clasificación como existencias dependería de la naturaleza del negocio de la compañía. Por ejemplo, si estamos evaluando los estados financieros de los exchangers, los cuales minan las monedas, incurriendo en un costo de producción, sus inventarios son los bitcoins, cerrando así su ciclo de producción al venderlos a un tercero, en conclusión, sólo si esta condición se cumple -que la obtención de bitcoins provenga del curso ordinario de producción- es válido incluirlas dentro de este renglón.

Activo intangible:

La NIC 38 (2004) Define un activo intangible como todo aquel "es un activo identificable, de carácter no monetario y sin apariencia física, que se posee para ser utilizado en la producción o suministro de bienes y servicios, para ser arrendado a terceros o para funciones relacionadas con la administración de la entidad. ".(p. 8) 
En este aspecto podemos mencionar, por ejemplo, que tanto las patentes o las marcas registradas, siempre han tenido una relación directa con el proceso productivo de la empresa, mientras que las criptomonedas no, en este sentido puede relacionarse a las actividades de la empresa en la medida que su valor incremente y genere beneficios extraordinarios, es como si la esencia del intangible existe porque no es un activo físico, pero en la práctica parece no tener la funcionalidad que reza la norma. Por último, en teoría los criptoactivos son "dinero", aunque por su parte muchos organismos públicos no lo reconocen así, incluso a sabiendas sirve para adquirir bienes o servicios inherentes a las empresas. En definitiva, parece que ésta es la clasificación más aproximada para contabilizarlo.

De este punto en adelante vendrán muy seguramente cambios sobre esta norma, porque lo cierto es que por los avances tecnológicos de nuestra era, ya las viejas metodologías demandan ser reemplazadas por otras que permitan la facilidad de incluirlas en los estados financieros, a fin de hacer más simple la tarea del auditor en los procesos de gestión empresarial, por esta razón los profesionales en las áreas de conocimiento relacionadas a las finanzas y contaduría prepararse para estar a la vanguardia, y enfrentar así los nuevos retos que impone la innovación monetaria y financiera.

\section{Bitcoin y la contabilidad en la auditoría de empresas}

Smart contracts como valor agregado al paradigma contractual:

Los smart contracts, contratos digitales o contratos inteligentes, son protocolos o programas informáticos que facilitan, verifican y hacen cumplir la negociación de un contrato programable en una plataforma digital la cual ejecuta el cumplimiento del mismo sin necesidad de una sentencia o ejecución legal. Los smart contracts, por su propia naturaleza, vienen a ser herramientas 
fundamentales que servirán para el desarrollo de las áreas contables, y con mayor énfasis en la auditoría, porque normalmente se componen de una interfaz de usuario donde se pueden emular la lógica de las cláusulas contractuales. Y en esta medida, cuando se dispara una condición preestablecida, el contrato inteligente ejecuta la cláusula contractual programada. En la práctica, tienen como objetivo brindar una seguridad superior a la ley de contrato tradicional y reducir costos de transacción asociados a la contratación de servicios empresariales. Permitiéndole a la auditoría garantizar la consecución de los procesos y los vínculos comerciales.

Éstas son algunas de las aplicaciones disponibles para los smarts contracts:

a) Prevención de incumplimiento: Este tipo de contrato se usa como instrumento de prevención, pues impide a quien adquirió una licencia, usarla fuera de los términos y condiciones establecidos, un ejemplo de uso de éste tipo de contratos es el caso de la copia de archivos de música, amparados por derechos de autor.

b) Traspaso de derechos de propiedad: En la obtención de bienes inmuebles, que funcionen bajo un sistema digital de propietarios, donde al comprobar que la transacción de compra fue ejecutada, automáticamente, y sin derecho a detenerla acredita al comprador la propiedad del mismo.

c) Herencias: En sentido se podría configurar el contrato inteligente, para que una vez verificado el fallecimiento de la persona, el smart contract se active, y acredite propiedades y dinero como debidamente fue programado.

d) Cuentas escrow: Este contrato inteligente haría las veces de intermediario, en este caso, el comprador de un bien o servicio realiza la cancelación del mismo a la cartera del proveedor y 
Dewis E. Alvarez-Pincay; Sandra P. Toala-Bozada; Zoila M. Delgado-Gutierrez; José F. Peñafiel-Loor; Adela del Jesus Lucio-Pillasagua; Viviana del Rocío Saltos-Buri

una vez que el smart contract comprueba que el objeto del intercambio ha llegado a su destino final (por ejemplo, usando tecnología GPS), de forma automática acredita el pago al vendedor.

El gran avance que ha logrado la tecnología ha brindado además una alternativa para llevar un proceso de auditoría menos engorroso, donde una aplicación posible podría realizarse sobre las unidades de negocio de un grupo de empresas, o sobre los departamentos, para velar que se cumplan los objetivos mínimos de calidad e ingresos pautados por la gerencia. En otros aspectos, ésta herramienta también puede ser usada para la realización de contratos proveedor-clienta, además puede ser útil para determinar el nivel de solvencia de una compañía respecto a sus obligaciones contraídas, tal y como lo ha logrado Deloitte hace poco.

\section{Primera compañía auditada mediante tecnología blockchain:}

Desde hace unos años, Deloitte ha estado desarrollando aplicaciones de la tecnología blockchain para comercializar servicios financieros (y de auditoría), y finalmente para principios de este año logró con éxito aplicar una prueba de solvencia a una compañía de gestión de criptomonedas y otros instrumentos, llamada ICONOMI, a este tipo de servicio se le conoce como auditoría blockchain, el cual consistió, en primera instancia, en desarrollar toda la programación de los pasivos, para así garantizar a los usuarios de ICONOMI los saldos de su plataforma. Luego se levantó un esquema con todos los balances, preservando el anonimato del usuario. Finalmente se aplicaron las reservas reales de la compañía a la base de datos de la cadena de bloques, para de esta forma poner a prueba el flujo de efectivo de la empresa en correspondencia a los balances, así como las cuentas bancarias en moneda convencional. 
El resultado final de la auditoría ofreció como resultado la garantía de solvencia de la plataforma, siendo esta última acreditada con proof of solvence (PoS) que avala la posesión de 80 activos digitales, un total de pasivos en el orden de los $\$ 133.6$ millones y reservas equivalente $\$ 210.2$ millones.

\section{Reflexion final:}

A este nivel investigativo podemos concluir con toda seguridad que la blockchain es una tecnología necesariamente revolucionaria, tanto, que para muchos entenderla a la perfección resulta un reto muy grande, donde las generaciones más anticuadas deberán seguir el ejemplo de ICONOMI, que ahora precisa ser un ente de confianza para sus usuarios actuales y futuros, es decir, la nueva versión 2.0 que ofrece la auditoría blockchain, busca limar las asperezas que deja la falta de ética en el manejo de la información, principalmente de las firmas de inversión y gestión de fondos de terceras partes.

Tras bastidores de este avance tecnológico, permanece bajo las sombras una discusión académica que tiene un alcance tan amplio como el propio cuestionamiento de las reglas que guían los negocios y los nuevos emprendimientos en ésta era digital. Respecto a ello, las empresas de auditoría forman parte del viejo sistema que está en discusión. Comenzando por los signos de obsolescencia que muestran las NIC por las cuales se rigen las técnicas de contabilidad y terminando por la necesidad firmas de auditorías que den un salto a la innovación, combinando lo mejor del mundo cibernético para la certificación pura y transparente del estado real de las empresas. En respuesta a las crisis económicas y éticas que acontecen por la falta de terceros de confianza, la auditoría debe tomar protagonismo y ser proactiva, renovar por completo los procesos, y formar a contadores altamente calificados en el área informática, tanto para la automatización de procesos, 
como la formación de auditores informáticos de alto nivel. Creo que a la luz de una solución que parece ser la panacea para las crisis de ética y transparencia a nivel mundial, por su parte la contabilidad está atravesando por una crisis académica, un reto que debe superar sobre las bases de una educación más especializada a las herramientas informáticas de última generación, en este sentido:

La Instituciones de Educación Superior tienen la brújula orientadora, por medio del diseño de Programas Académicos para formar contables con criterio científico, capaces de afrontar la crisis disciplinal y hacer frente a los retos, que impone la globalización, preparándolos para atender la problemática que se cierne sobre la sociedad del conocimiento la cual puede tener los comandos de control por medio de la Contabilidad. (Rojas, 2012 p.19)

Develar esta realidad es a lo que muchos les causan tanto pánico como escepticismo, pero lo cierto del caso es que las grandes compañías, como Deloitte o Amazon, están avanzando en esta materia y con el tiempo la brecha se irá ampliando más. Es decir, los sistemas de valores y los paradigmas sociales están cambiando en función de una tecnología que se mueve a una velocidad progresiva, de manera que suponer que la auditoría es infalible a este rezago sería correr el riesgo de convertirnos en una comunidad académica prescindible.

\section{Bibliografia.}

Angulo, J. A. (2004). Instrumentos Financieros. En J. L. Álvarez, S. d. Valdelomar, L. C. Calvo, \& J. A. Angulo, Monografías sobre las normas internacionales de información financiera (pág. 598). Madrid: Recoletos.

Bargiela, J. H. (2016). ¿cómo puede una empresa argentina operar y tomar deuda en criptomonedas, registrando su contabilidad de una forma transparente y asegurar el cumplimiento de la ley al mismo tiempo? Argentina: Universidad Torcuato Di Tella.

Echebarria, M. (2017). Contratos electrónicos autoejecutables (smart contract), y pagos con tecnología blockchain. Revista de Estudios Europeos, 69-97. 
Kam, Y. (Marzo de 2017). Descifrando el código de la criptomoneda, ¿qué es un bitcoin? Noticias NIIF PWC, pág. 9.

Karp, N. (2017). Tecnología de cadena de bloques (blockchain): la última disrupción en el sistema financiero. BBVA research.

Menéndez, J. P., \& Pérez, B. Á. (23 de Febrero de 2017). Registro Contable y Marco Normativo de las Criptomonedas. Recuperado el 15 de Mayo de 2018, de fundacionctico: https://www.fundacionctic.org/sites/default/files/files/UniOviJornada23M\%20\%20MarcoNorm\%26RC.pdf

Molina, D. I. (2016). Fintech: Lo que la tecnología hace por las finanzas. Profit Editorial.

Normas Internacionales de Contabilidad (NIC). (s.f.). Obtenido de http://www.normasinternacionalesdecontabilidad.es/nic/nic.htm

Preukschat, A. (. (2017). Blockchain: La revolución industrial de internet. Editorial Gestión 2000.

Remolina, N. (2017). La incorporación del Blockchain en el Derecho de sociedades de Delaware. Derecho y finanzas.

Rojas, S. B. (2012). GLOBALIZACIÓN Y EDUCACION CONTABLE, CRISIS Y RETOS. IV Encuentro nacional de profesores de contaduria pública. Bogotá: Universidad Libre.

Sierra, M. (Noviembre de 2017). ¿Como Contabilizar las Monedas Digitales: Criptomonedas o Dinero Digital? Recuperado el 15 de Mayo de 2018, de Steemit: https://steemit.com

Viloria, C. (2017). banca y negocios. Recuperado el 15 de Mayo de 2018, de banca y negocios: http://www.bancaynegocios.com 